\title{
Solution of Nonlinear Integro Differential Equations by Two-Step Adomian Decomposition Method (TSAM)
}

\author{
Maryam Al-Mazmumy¹, Safa 0. Almuhalbedi ${ }^{2}$ \\ ${ }^{1}$ Department of Mathematics, Faculty of Science-Al Faisaliah Campus, King Abdulaziz University, Jeddah, Saudi Arabia \\ ${ }^{2}$ Department of Mathematics, Faculty of Science, King Abdulaziz University, Jeddah, Saudi Arabia \\ Email:malmazmumy@kau.edu.sa,sss-2011-s@hotmail.com
}

How to cite this paper: Al-Mazmumy, M. and Almuhalbedi, S.O. (2016) Solution of Nonlinear Integro Differential Equations by Two-Step Adomian Decomposition Method (TSAM). International Journal of Modern Nonlinear Theory and Application, 5, 248255.

http://dx.doi.org/10.4236/ijmnta.2016.54022

Received: November 4, 2016

Accepted: December 13, 2016

Published: December 16, 2016

Copyright $\odot 2016$ by authors and Scientific Research Publishing Inc. This work is licensed under the Creative Commons Attribution International License (CC BY 4.0).

http://creativecommons.org/licenses/by/4.0/

\begin{abstract}
The Adomian decomposition method (ADM) can be used to solve a wide range of problems and usually gets the solution in a series form. In this paper, we propose two-step Adomian Decomposition Method (TSAM) for nonlinear integro-differential equations that will facilitate the calculations. In this modification, compared to the standard Adomian decomposition method, the size of calculations was reduced. This modification also avoids computing Adomian polynomials. Numerical results are given to show the efficiency and performance of this method.
\end{abstract}

\section{Keywords}

Adomian Decomposition Method, Nonlinear Volterraintegro-Differential Equations, Nonlinear Fredholmintegro-Differential Equations, Two-Step

\section{Introduction}

In 1999, Wazwaz [1] presented a powerful modification to the "Adomian Decomposition Method" (ADM) that accelerated the rapid convergence of the series solution as compared with the standard Adomian method [2]. The modified technique has been shown to be computationally efficient while applied to several important differential and integral equations in the research. In all cases of applied fields, excellent performance is obtained that may lead to a widespread application in many applied sciences. In addition, the modified technique may give the exact solution for nonlinear equation without any need of the so-called Adomian polynomials [3].

In spite of the fact that the "Modified Decomposition Method" of wazwaz has shown to be computationally efficient in some applications, the criterion of separating the 
function $\varnothing$ into two appropriate parts $\varnothing_{1}$ and $\varnothing_{2}$, and when the function $\varnothing$ includes only one term, this case remains unsolved. Furthermore, the "Modified Decomposition Method" does not always minimize the required size of calculations, and often needs more computation than the common Adomian method. In 2005, X.G. Luo [4] proposed the "Two-Step Adomian Decomposition Method" (TSADM) as a modification to the common "Adomian Decomposition Method". The TSADM may provide the solution by using a single iteration only and reduces the quantity of computation compared with the common "Adomian Decomposition Method" and the modified method. The two-Step decomposition method perhaps also produces the exact solution without any requirement of the polynomials of Adomian. In 2006, X.G. Lou et al. [5] showed by experimentation that the TSADM extended to solve systems of inhomogeneous equations. Several researchers applied this modification for solving a huge class of problems, such as: in 2008, D.N. Khan, et al. [6] used the TSADM to solve the heat equation, in 2015, M. Al-Mazmumy, et al. [7] used this modification for nonlinear partial differential equation and in 2013, H. O. Bakodah [8] used the TSADM for solving the nonlinear Abel's Integral equation. In this paper, we use the (TSADM) to obtain the solutions of the integro-differential equations and the system of integro differential equations. Wide classes of nonlinear integro-differential equations, both Volterra as well as Fredholm, can be solved by the (TSADM). This paper is organized as follows. In Section 2, it is shown the principles of the standard Adomian method and the analysis of the proposed method is given. In Section 3, a comparative study between TSADM and previous methods is illustrated with the help of several examples. Concluding remarks follow in Section 4 .

\section{Description of the Method (TSADM)}

We consider the Integro-differentil equation of the form

$$
u^{\prime \prime}(x)=f(x)+\int_{a}^{b(x)} k(x, t) \cdot(l u(t)+N(u(t))) \mathrm{d} t
$$

with initial condotions $u(0)=\alpha, u^{\prime}(0)=\beta$.

Where $u^{\prime \prime}(x)=\frac{\mathrm{d}^{2} u}{\mathrm{~d} x^{2}}$ is the second derivative of the unknown function $u(x)$ that will be determined, $k(x, t)$ are the kernels of the integro differential equations, $f(x)$ are an analytic function, $a$ and $b(x)$ are the limits of integration may be both constants or mixed. And $l u(t), N(u(t))$ are linear and nonlinear term, respectively.

Let $L=\frac{\mathrm{d}^{2}}{\mathrm{~d} x^{2}}$, so $L^{-1}()=.\int_{0}^{x} \int_{0}^{x}(.) \mathrm{d} x \mathrm{~d} x$, applying $L^{-1}$ to both sides of $(1)$, and using initial conditions, we obtain

$$
u(x)=\alpha+\beta x+L^{-1} f(x)+L^{-1} \int_{a}^{b(x)} k(x, t) \cdot(l u(t)+N(u(t))) \mathrm{d} t
$$

For nonlinear equations, the nonlinear operator $N(u)=F(u)$ is usually represented by an infinite series of the Adomian polynomials

$$
F(u)=\sum_{n=0}^{\infty} A_{n}
$$


The standard Adomian method defines the solution $u$ by the series

$$
u=\sum_{n=0}^{\infty} u_{n}
$$

where the components $u_{0}, u_{1}, u_{2}, \cdots$ are usually determined recursively by:

$$
\left\{\begin{array}{l}
u_{0}=\alpha+\beta x+L^{-1} f(x) \\
u_{k+1}=L^{-1} \int_{a}^{b(x)} k(x, t) \cdot\left(u_{k}+A_{k}\right) \mathrm{d} t, k \geq 1
\end{array}\right.
$$

The main ideas of the proposed "Two-Step Adomian Decomposition Method" are:

(1) Applying the inverse operator $L^{-1}$ to $f$, and using the given conditions it is obtained:

$$
\varphi=\varnothing+L^{-1} f
$$

where the function $\varnothing$ represents the terms arising from using the given conditions. To achieve the objectives of this method, it is set:

$$
\varphi=\varphi_{0}+\varphi_{1}+\cdots+\varphi_{m},
$$

where $\varphi_{0}, \varphi_{1}, \cdots, \varphi_{m}$ are the terms arising from integrating $f$ and from using the given conditions. Based on this, the function $u_{o}$ is defined as:

$$
u_{0}=\varphi_{k}+\cdots+\varphi_{k+s}
$$

where $k=0,1, \cdots, m, s=0,1, \cdots, m-k$. Then, by substitution, verify that $u_{0}$ satisfies the integro differential equation (1) and the given conditions. Once the exact solution is obtained, the process is ended, otherwise, go to the following step two.

(2) We set $u_{0}=\varphi$ and continue with the standard Adomian recursive relation

$$
u_{k+1}=L^{-1} \int_{a}^{b(x)} k(x, t) \cdot\left(u_{k}+A_{k}\right) \mathrm{d} t, k \geq 0
$$

Compared to the common "Adomian Decomposition Method" and the "Modified Decomposition Method", it is clear that the "Two-Step Decomposition Method" may produce the solution by using only one iteration. It is worthy to note that the Procedure of verification in the first step can be larg effective in many cases. This can be note through the following examples. Further, the "Two-Step Decomposition Method" avoids the difficulties arising in the modified method. Also the number of the terms in $\varphi$, namely $m$, is small in many practical problems.

\section{Computational Results and Analysis}

\section{Example 1}

Consider nonlinear Volterraintegro-differential equation [9]

$$
u^{\prime}(x)=1-\frac{1}{2} x+\frac{x \mathrm{e}^{-x^{2}}}{2}+\int_{0}^{x} x t \cdot \mathrm{e}^{-u^{2}(t)} \mathrm{d} t, u(0)=0
$$

With the exact solution is $u(x)=x$. Applying $L^{-1}()=.\int_{0}^{x}(.) \mathrm{d} x$ in both sides given,

$$
u(x)=x-\frac{1}{4} x^{2}+\frac{1}{4}-\frac{1}{4} \mathrm{e}^{-x^{2}}+L^{-1} \int_{0}^{x} x t \cdot \mathrm{e}^{-u^{2}(t)} \mathrm{d} t
$$

The modified decomposition method: Using the modified recursive relation (10), and by selecting $u_{0}=\varnothing_{1}=x$, we obtain 


$$
u_{1}=-\frac{1}{4} x^{2}+\frac{1}{4}-\frac{1}{4} \mathrm{e}^{-x^{2}}+L^{-1} \int_{0}^{x} x t \cdot \mathrm{e}^{-u_{0}^{2}(t)} \mathrm{d} t=0
$$

In view of (12), the exact solution is given by $u(x)=x$.

It is to be noted that if we select $u_{0}=\varnothing_{2}=-\frac{1}{4} x^{2}+\frac{1}{4}-\frac{1}{4} \mathrm{e}^{-x^{2}}$, the same size of computational work required compared to the standard Adomian method.

The (TSADM), using the scheme (7) gives

$$
\varphi=\varphi_{0}+\varphi_{1}+\varphi_{2}+\varphi_{3}, \quad \varphi_{0}=x, \quad \varphi_{1}=-\frac{1}{4} x^{2}, \quad \varphi_{2}=\frac{1}{4}, \varphi_{3}=-\frac{1}{4} \mathrm{e}^{-x^{2}} .
$$

By selecting $u_{0}=\varphi_{0}$, and by verifying that $u_{0}$ justifies equation (10) and the given initial condition, the same solution is obtained immediately $u(x)=x$

However, we use the standard Adomian method to find:

$$
\left\{\begin{array}{c}
u_{0}=x-\frac{1}{4} x^{2}+\frac{1}{4}-\frac{1}{4} \mathrm{e}^{-x^{2}} \\
u_{1}=L^{-1} \int_{0}^{x} x t \cdot A_{0} \mathrm{~d} t \\
u_{2}=L^{-1} \int_{0}^{x} x t \cdot A_{1} \mathrm{~d} t \\
\vdots
\end{array} .\right.
$$

In view of (14), the modified method also requires a huge size of computational work to obtain few terms of the series. Moreover, the same as the standard Adomian decomposition method, the modified method requires the use of the Adomian polynomials for nonlinear models. However, using the two-step Adomian decomposition method, there is no need to use the Adomian polynomials.

\section{Example 2}

Consider nonlinear Fredholmintegro-differential equation

$$
u^{\prime \prime}(x)=\mathrm{e}^{x}-1+\int_{0}^{1} \mathrm{e}^{-4 t} u^{2}(t)\left(u^{\prime}(t)\right)^{2} \mathrm{~d} t, u(0)=u^{\prime}(0)=1
$$

With the exact solution is $u(x)=\mathrm{e}^{x}$.

Applying $L^{-1}()=.\int_{0}^{x}(.) \int_{0}^{x}(.) \mathrm{d} x \mathrm{~d} x$ in both sides given,

$$
u(x)=\mathrm{e}^{x}-\frac{1}{2} x^{2}+L^{-1} \int_{0}^{1} \mathrm{e}^{-4 t} u^{2}(t)\left(u^{\prime}(t)\right)^{2} \mathrm{~d} t
$$

The modified decomposition method: Using the modified recursive relation (15), and by selecting $u_{0}=\varnothing_{1}=\mathrm{e}^{x}$, we obtain

$$
u_{1}=-\frac{1}{2} x^{2}+L^{-1} \int_{0}^{1} \mathrm{e}^{-4 t} \cdot u_{0}^{2} u_{0}^{\prime 2} \mathrm{~d} t=0
$$

In view of (17), the exact solution is given by $u(x)=\mathrm{e}^{x}$.

It is to be noted that if we select $u_{0}=\varnothing_{2}=-\frac{1}{2} x^{2}$, the same size of computational work required compared to the standard Adomian method.

The (TSADM), using the scheme (7) gives

$$
\varphi=\varphi_{0}+\varphi_{1} \varphi_{0}=\mathrm{e}^{x}, \varphi_{1}=-\frac{1}{2} x^{2}
$$


By selecting $u_{0}=\varphi_{0}$, and by verifying that $u_{0}$ justifies equation (15) and the given initial condition, the same solution is obtained immediately $u(x)=\mathrm{e}^{x}$.

However, we use the standard Adomian method to find:

$$
\left\{\begin{array}{c}
u_{0}=\mathrm{e}^{x}-\frac{1}{2} x^{2} \\
u_{1}=L^{-1} \int_{0}^{1} \mathrm{e}^{-4 t} \cdot A_{0} \mathrm{~d} t \\
u_{2}=L^{-1} \int_{0}^{1} \mathrm{e}^{-4 t} \cdot A_{1} \mathrm{~d} t \\
\vdots
\end{array}\right.
$$

In view of (19), the modified method also requires a huge size of computational work to obtain few terms of the series. Moreover, the same as the standard Adomian decomposition method, the modified method requires the use of the Adomian polynomials for nonlinear models. However, using the two-step Adomian decomposition method, there is no need to use the Adomian polynomials.

\section{Example 3}

Consider the system of nonlinear Volterraintegro differential equation [10]

$$
\left\{\begin{array}{l}
u^{\prime \prime}(x)=-\sin (x)-\cos (x)+\int_{0}^{x} \cos (x+t)\left(u^{2}(t)+v^{2}(t)\right) \mathrm{d} t, u(0)=1, u^{\prime}(0)=0 \\
v^{\prime \prime}(x)=-\sin (x)-\frac{1}{2} \sin (2 x)+\int_{0}^{x}\left(u^{2}(t)-v^{2}(t)\right) \mathrm{d} t, v(0)=0, v^{\prime}(0)=1
\end{array}\right.
$$

With the exact solution are $(\cos (x), \sin (x))$.

Applying $L^{-1}()=.\int_{0}^{x} \int_{0}^{x}(.) \mathrm{d} x \mathrm{~d} x$ of both sides gives

$$
\left\{\begin{array}{l}
u(x)=\sin (x)-x+\cos (x)+L^{-1} \int_{0}^{x} \cos (x+t)\left(u^{2}(t)+v^{2}(t)\right) \mathrm{d} t \\
v(x)=\sin (x)+\frac{1}{8} \sin (2 x)-\frac{1}{4} x+L^{-1} \int_{0}^{x}\left(u^{2}(t)-v^{2}(t)\right) \mathrm{d} t
\end{array}\right.
$$

The modified decomposition method: Using the modified recursive relation (20), and by selecting $u_{0}=\varnothing_{1_{1}}=\cos (x)$ and $v_{0}=\varnothing_{2_{1}}=\sin (x)$, we obtain

$$
\left\{\begin{array}{l}
u_{1}=\sin (x)-x+L^{-1} \int_{0}^{x} \cos (x+t)\left(u_{0}^{2}(t)+v_{0}^{2}(t)\right) \mathrm{d} t=0 \\
v_{1}=\frac{1}{8} \sin (2 x)-\frac{1}{4} x+L^{-1} \int_{0}^{x}\left(u_{0}^{2}(t)-v_{0}^{2}(t)\right) \mathrm{d} t=0
\end{array}\right.
$$

In view of (22), the exact solution is given by $(u(x), v(x))=(\cos (x), \sin (x))$.

It is to be noted that if we select

$u_{0}=\varnothing_{1_{2}}=\sin (x)-x$ and $v_{0}=\varnothing_{2_{2}}=\frac{1}{8} \sin (2 x)-\frac{1}{4} x$, the same size of computational work required compared to the standard Adomian method.

The (TSADM), using the scheme (7) gives

$$
\begin{gathered}
\left\{\begin{array}{l}
\varphi=\varphi_{0}+\varphi_{1}+\varphi_{2} \\
\mu=\mu_{0}+\mu_{1}+\mu_{2}
\end{array}\right. \\
\left\{\begin{array}{l}
\varphi_{0}=\sin (x), \varphi_{1}=-x, \varphi_{2}=\cos (x) \\
\mu_{0}=\sin (x), \mu_{1}=\frac{1}{8} \sin (2 x), \mu_{2}=-\frac{1}{4} x
\end{array}\right.
\end{gathered}
$$


By selecting

$$
\left\{\begin{array}{l}
u_{0}=\cos (x) \\
v_{0}=\sin (x)
\end{array}\right.
$$

and by verifying that $u_{0}, v_{0}$ justifies equation (20) and the given intial conditions, the same solution is obtained immediately.

$$
\left\{\begin{array}{l}
u(x)=\cos (x) \\
v(x)=\sin (x)
\end{array}\right.
$$

However, we use the standard Adomian method to find:

$$
\begin{gathered}
\left\{\begin{array}{c}
u_{0}=\sin (x)-x+\cos (x) \\
v_{0}=\sin (x)+\frac{1}{8} \sin (2 x)-\frac{1}{4} x \\
\vdots
\end{array}\right. \\
\begin{cases}u_{k+1}=L^{-1} \int_{0}^{x} \cos (x+t)\left(A_{k}+B_{k}\right), & k \geq 0 \\
v_{k+1}=L^{-1} \int_{0}^{x}\left(A_{k}-B_{k}\right), & k \geq 0\end{cases}
\end{gathered}
$$

In view of (26), the modified method also requires a huge size of computational work to obtain few terms of the series. Moreover, the same as the standard Adomian decomposition method, the modified method requires the use of the Adomian polynomials for nonlinear models. However, using the two-step Adomian decomposition method, there is no need to use the Adomian polynomials.

Example 4

Consider the system of nonlinear Fredholmintegro-differential equation [10]

$$
\left\{\begin{array}{l}
u^{\prime}(x)=\sin (x)+x \cos (x)-\frac{\pi^{3}}{3}+\int_{0}^{\pi}\left(u^{2}(t)+v^{2}(t)\right) \mathrm{d} t, u(0)=0 \\
v^{\prime}(x)=\cos (x)-x \sin (x)+\frac{\pi}{2}+\int_{0}^{\pi}\left(u^{2}(t)-v^{2}(t)\right) \mathrm{d} t, v(0)=0
\end{array}\right.
$$

With exact solution $(x \sin (x), x \cos (x))$. Applying $L^{-1}()=.\int_{0}^{x}(.) \mathrm{d} x$ of both sides gives

$$
\left\{\begin{array}{l}
u(x)=x \sin (x)-\frac{\pi^{3}}{3} x+L^{-1} \int_{0}^{\pi}\left(u^{2}(t)+v^{2}(t)\right) \mathrm{d} t \\
v(x)=x \cos (x)+\frac{\pi}{2} x+L^{-1} \int_{0}^{\pi}\left(u^{2}(t)-v^{2}(t)\right) \mathrm{d} t
\end{array}\right.
$$

The modified decomposition method: Using the modified recursive relation (27), and by selecting $u_{0}=\varnothing_{1_{1}}=x \sin (x)$ and $v_{0}=\varnothing_{2_{1}}=x \cos (x)$, we obtain

$$
\left\{\begin{array}{l}
u_{1}=-\frac{\pi^{3}}{3} x+L^{-1} \int_{0}^{\pi}\left(u_{0}^{2}(t)+v_{0}^{2}(t)\right) \mathrm{d} t=0 \\
v_{1}=\frac{\pi}{2} x+L^{-1} \int_{0}^{\pi}\left(u_{0}^{2}(t)-v_{0}^{2}(t)\right) \mathrm{d} t=0
\end{array}\right.
$$

In view of (29), the exact solution is given by $(u(x), v(x))=(x \sin (x), x \cos (x))$. 
It is to be noted that if we select $u_{0}=\varnothing_{1_{2}}=-\frac{\pi^{3}}{3} x$ and $v_{0}=\varnothing_{2_{2}}=\frac{\pi}{2} x$, the same size of computational work required compared to the standard Adomian method.

The (TSADM), using the scheme (7) gives

$$
\begin{gathered}
\left\{\begin{array}{l}
\varphi=\varphi_{0}+\varphi_{1} \\
\mu=\mu_{0}+\mu_{1}
\end{array}\right. \\
\left\{\begin{array}{l}
\varphi_{0}=x \sin (x), \quad \varphi_{1}=-\frac{\pi^{3}}{3} x \\
\mu_{0}=x \cos (x), \quad \mu_{1}=\frac{\pi}{2} x
\end{array}\right.
\end{gathered}
$$

By selecting

$$
\left\{\begin{array}{l}
u_{0}=x \sin (x) \\
v_{0}=x \cos (x)
\end{array}\right.
$$

and by verifying that $u_{0}, v_{0}$ justifies equation (27) and the given initial conditions, the same solution is obtained immediately.

$$
\left\{\begin{array}{l}
u(x)=x \sin (x) \\
v(x)=x \cos (x)
\end{array}\right.
$$

However, we use the standard Adomian method to find:

$$
\begin{gathered}
\left\{\begin{array}{c}
u_{0}=x \sin (x)-\frac{\pi^{3}}{3} x \\
v_{0}=x \cos (x)+\frac{\pi}{2} x \\
\vdots
\end{array}\right. \\
\left\{\begin{array}{c}
u_{k+1}=L^{-1} \int_{0}^{\pi}\left(A_{k}+B_{k}\right), k \geq 0 \\
v_{k+1}=L^{-1} \int_{0}^{\pi}\left(A_{k}-B_{k}\right), k \geq 0
\end{array}\right.
\end{gathered}
$$

In view of (33), the modified method also requires a huge size of computational work to obtain few terms of the series. Moreover, the same as the standard Adomian decomposition method, the modified method requires the use of the Adomian polynomials for nonlinear models. However, using the two-step Adomian decomposition method, there is no need to use the Adomian polynomials.

\section{Conclusion}

In this paper, we have applied two-step Adomian Decomposition Method (TSAM) to obtain the solutions of nonlinear integro-differential equations. Some examples have been discussed as illustrations. In this work, we show that TSADM is convenient to solve integro-differential equations and reduce the size of calculations compared to the standard Adomian decomposition method and modified decomposition method. This modification also avoids computing Adomian polynomials. The TSADM produce the 
solution by using only two iterations, if compared with the common Adomian method and the modified method. Moreover, the TSADM overcomes the difficulties arising in the modified decomposition method.

\section{References}

[1] Wazwaz, A. (1999) A Reliable Modification of Adomian Decomposition Method. Applied Mathematics and Computation, 102, 77-86. https://doi.org/10.1016/S0096-3003(98)10024-3

[2] Adomian, G. (1994) Solving Frontier Problems of Physics: The Decomposition Method. Kluwer Academic Publisher, Boston. https://doi.org/10.1007/978-94-015-8289-6

[3] Rach, R. (2008) A New Definition of the Adomian Polynomials. Kybernetes, 37, 910-955. https://doi.org/10.1108/03684920810884342

[4] Luo, X.G. (2005) A Two-Step Adomian Decomposition Method. Applied Mathematics and Computation, 170, 570-583. https://doi.org/10.1016/j.amc.2004.12.010

[5] Zhang, B.Q., Wu, Q.B. and Luo, X.G. (2006) Experimentation with Two-Step Adomian Decomposition Method to Solve Evolution Models. Applied Mathematics and Computation, 175, 1495-1502.

[6] Khan Marwat, D.N. and Asghar, S. (2008) Solution of the Heat Equation with Variable Properties by Two-Step Adomian Decomposition Method. Mathematical and Computer Modelling, 48, 83-90. https://doi.org/10.1016/j.mcm.2007.09.003

[7] Al-Mazmumy, M. and Al-Malki, H. (2015) Some Modification of Adomian Decomposition Method for Nonlinear Partial Differential Equations. IJRAS, 23.

[8] Bakodah, H.O. (2013)Adomian Decomposition Method and Its Modification for Nonlinear Abel's Integral Equation. International Journal of Mathematical Analysis, 48, 2349-2358.

[9] Dehghan, M. and Salehi, R. (2012) The Numerical Solution of the Non-Linear Integro-Differential Equations Based on the Meshless Method. Journal of Computational and Applied Mathematics, 236, 2367-2377. https://doi.org/10.1016/j.cam.2011.11.022

[10] Wazwaz, A.M. (2011) Linear and Nonlinear Integral Equations: Methods and Applications. Springer-Verlag, Beijing. https://doi.org/10.1007/978-3-642-21449-3

Submit or recommend next manuscript to SCIRP and we will provide best service for you:

Accepting pre-submission inquiries through Email, Facebook, LinkedIn, Twitter, etc.

A wide selection of journals (inclusive of 9 subjects, more than 200 journals)

Providing 24-hour high-quality service

User-friendly online submission system

Fair and swift peer-review system

Efficient typesetting and proofreading procedure

Display of the result of downloads and visits, as well as the number of cited articles

Maximum dissemination of your research work

Submit your manuscript at: http://papersubmission.scirp.org/

Or contact ijmnta@scirp.org 